\title{
Care Seeking Behavior of Citizens During Pandemics: A Case Study of COVID-19 in Nigeria
}

\section{B Tijani, o S Amoo*, T Adaramewa, D N Kareithi, A Omavuohrerhe, $S$ Karera and B L Salako}

Nigerian Institute of Medical Research, NIMR, Nigeria

*Corresponding Author: O S Amoo, Nigerian Institute of Medical Research, NIMR, Nigeria.
Received: June 19, 2020

Published: August 31, 2020

(C) All rights are reserved by $\mathbf{O} \mathbf{S}$ Amoo., et

al.

\begin{abstract}
The COVID-19 virus is rapidly crossing borders and spreading across countries and the globe, with 212 countries currently affected as of May 2020, 6 months since reports of the first case in November 2019 in Wuhan, China. Global efforts to lessen the impact of COVID-19 pandemic span across surveillance, preparedness, response, prevention, testing, contact tracing, and treatment. Co-creation Hub (CcHUB) in collaboration with the Nigerian Institute of Medical Research (NIMR) and LifeBank developed a digital system to support the process of free testing of suspected COVID-19 cases to respond to the pandemic in Nigeria and deployed it in Lagos. An average of $25 \%$ of suspected cases presented for testing after scheduling appointments, meaning only a fourth of the highrisk cases as defined by the system were tested, revealing a drop-out rate of $74 \%$. Preliminary analysis seeking to check which factors are associated with patients who presented themselves for testing yielded no significant factors, forming the rationale for our study.

The study was a cross sectional study, seeking to 1) Understand determinants of healthcare seeking behaviors and healthcare utilization during a pandemic, 2) Provide information and guide health planners, administrators and policy makers on factors that enhance effective utilization of structures and platforms for service delivery. The variables collected were: demography and socioeconomic status, health status, satisfaction with healthcare system, satisfaction with NIMR service, mode of transport used/to be used and general feedback.

Data collection was done using a close-ended survey and social media analysis - analysing twitter sentiments around COVID-19 testing in Nigeria. 300 study participants from a target population of 566 were selected using a simple random sampling for a finite population. Descriptive statistics and analysis looking for association (odds ratios (OR) with 95\% confidence interval (CI), including bivariate and multivariate unconditional logistic regression) were conducted using STATA 16.

Our results show that distance to facility, preexisting health conditions, knowledge of COVID-19 symptoms, previous healthcare experience, type of and frequency of communication influence healthcare seeking behavior of Nigerians, specifically during COVID-19. We recommend that the strategic placement of testing centers and increasing knowledge of disease could enhance service utilization.
\end{abstract}

Keywords: COVID-19; Degree of Freedom; World Health Organization (WHO)

\section{Abbreviations}

API: Application Programming Interface; CCHUB: Co-Creation Hub Nigeria; CDC: Centers for Disease Control and Prevention; CHSQ: Chi-Square; C.I: Confidence Interval; COVID-19: Coronavirus Disease 2019; Df: Degree of Freedom; HIV: Human Immunodeficiency
Virus; LGA: Local Government Area; NCDC: Nigeria Centre for Disease Control; NIMR: Nigerian Institute of Medical Research; OR: Odd Ratio; PII: Personally Identifiable Information; SARS: Severe Acute Respiratory Syndrome; T: Hypothesis Test Statistics; TB: Tuberculosis; USA: The United States of America; WHO: World Health Organization 


\section{Background}

The COVID-19 virus is rapidly crossing borders and spreading across countries and the entire globe, with 212 countries currently affected as of May 2020, 6 months since reports of the first case in November 2019 in Wuhan, China. Different regions and continents have reported highly variable incidence of COVID-19 cases. The African continent till date has reported some of the lowest numbers [1]. Although there is no proven vaccine or medicine to prevent or treat, global efforts to lessen the impact of COVID-19 pandemic span across a wide range, - surveillance, preparedness, response, prevention, early identification, testing, contact tracing, and treatment.

Co-creation Hub (CcHUB) in collaboration with the Nigeria Institute of Medical Research (NIMR) and LifeBank developed a digital system to support the process of free testing for suspected COVID-19 cases to respond to the pandemic in Nigeria. The system supports the identification, reporting, triaging, testing of cases and communication of test results. The tool was first deployed in Lagos state- the epicenter in Nigeria.

The system was launched on $28^{\text {th }}$ March 2020, and was designed to retrieve information on demography, symptoms, pre-existing conditions, recent contact and travel history. The form was made openly accessible online and rolled out for use by the general public as seen on table 1 below. This system helps streamline all requests to ensure that high risk cases are prioritized for testing. Also, given the limited resources, an appointment system for testing was instituted so as to ensure measures of social distancing and other safety protocols are adhered to at the testing center.

Detecting high risk cases followed a 2-tier method: (i) a pre-set system of filtering symptoms and history of exposure (ii) backend validation (validation of personal details, review of symptoms and contact history etc). Cases that fit the high-risk criteria automatically received communication, usually within 24 - 48 hours after registration. Emails with details of scheduled appointments for testing were first sent, followed by phone calls to confirm appointments. Patients were required to present themselves to the NIMR drive-through testing site on scheduled days (Table 1).

\section{Literature Review}

"People use health-care services to diagnose, cure, or ameliorate disease or injury; to improve or maintain function; or to ob- tain information about their health status and prognosis." In theory, health-care utilization should correlate highly with the need, however, this is not always the case. Barriers to health-care utilization can be explained by several factors such as- age, gender, self-rated health, perceived quality of care, lack of trust in the system, knowledge of need for care, access to care, socioeconomic status, lack of insurance coverage, and cultural beliefs [2].

\begin{tabular}{|l|c|}
\hline Activity & Date \\
\hline Conception & $21 / 3 / 2020$ \\
\hline Form published first version to staging server & $24 / 3 / 2020$ \\
\hline $\begin{array}{l}\text { Mobile dashboard first published on staging } \\
\text { server }\end{array}$ & $25 / 3 / 2020$ \\
\hline Published on Live server & $27 / 3 / 2020$ \\
\hline Announced/Launched by NIMR & $28 / 3 / 2020$ \\
\hline First Submission & $28 / 3 / 2020$ \\
\hline Behavioral research & $9 / 4 / 2020$ \\
\hline
\end{tabular}

Table 1: Timeline of the online form.

Free health care services may improve discrepancy between potential and actual utilization of care. However, eliminating financial barriers to health services does not guarantee equitable healthcare distribution; positive buffers such as transportation and communication infrastructure may enable individuals to seek medical services $[3,4]$. In addition, more attention should be given to supply-side factors, such as quality of care, equipment and infrastructure, human resource for health, to ensure that improved access translates into better utilization and improved health outcomes [5].

\section{Rationale}

A total of 299 registrations, with a daily average of 60 registrations, was recorded on the system within the first 72 hours $\left(28^{\text {th }}\right.$ - $30^{\text {th }}$ March 2020) following its roll-out, which coincided with the period just before the commencement of the lockdown in Lagos state ( $31^{\text {st }}$ March 2020). However, these numbers began to dwindle in the following days, bringing the daily average to 48 registration for the month of April (a 20\% decrease). On the other hand, the number of suspected cases that presented for testing after scheduling appointments also remained below 30\% (29\% at the end of March, 26\% by the end of April). This implied that an average of $25 \%$ of the high-risk cases as defined by the system presented and were tested, revealing a drop-out rate of $74 \%$. 
We followed up on a subset of the high risk cases that did not present for testing and majority listed "it was a false alarm" (16\%) and "they had not seen the email" (11\%), as the top reasons for the no-show. Further investigation sought to check which factors are associated with patients who presented themselves for testing. The dependent variable was binary i.e. $1=$ showed up for testing, $0=$ did not show up for testing.

We began by conducting bivariate analyses to check which factors (gender, age, state of residence, nationality, marital status, confirmation of email sent and the symptoms listed during application) were individually associated with not showing up for testing. Univariate binary logistic regression was used and the bivariate analysis showed that none of the factors we gave were significantly associated with presenting oneself for testing at $\propto=0.05$.

These preliminary findings formed the rationale for our study. We sought to investigate the care seeking behaviour/determinants of health care utilization during a pandemic, with a focus on COVID-19 in Nigeria. The findings of this research will provide information to relevant stakeholders involved in healthcare service delivery on how to promote uptake of pandemic-related health interventions.

\section{Objectives of the Study}

This study sought to:

- Understand determinants of care seeking behaviours/determinants of health care utilization during a pandemic

- Provide information and guide health planners, administrators and policy makers on factors that enhance effective utilization of structures and platforms for service delivery

\section{Methodology}

Study design

A cross sectional study design was adopted for this research. A cross-sectional study examines the relationship between disease (or other health related state) and other variables of interest as they exist in a defined population at a single point in time or over a short period of time [6].

\section{Study population}

The selection for the study was: 1) All participants that had been invited to test after filling the Health Information form 2) All participants invited with a valid phone number.
Study area

The study was conducted in Nigeria.

Variables collected

The variables collected in the study were:

Outcome variable: presented for testing (binary, $1=$ Yes and $0=$ No)

- Demographic and socioeconomic variables

- $\quad$ Age, gender, marital status, education level, state and LGA of residence, employment status

- Health status variables

- Pre-existing condition

- $\quad$ Awareness about COVID-19 symptoms

- Source of health information

- Use of social media

- $\quad$ Satisfaction with healthcare system variables

- Communication

- Waiting time

- $\quad$ Treatment received

- Cleanness

- $\quad$ Satisfaction with NIMR service

- $\quad$ Process of filling the online form

- Communication after filling the form

- Clear instructions during the testing process

- Motivation to come for the testing

- Recommendations

- Challenges faced during the testing experience

- $\quad$ Mode of transport

- Mode of transport used for individuals who have been tested

- Mode of transport will be used for those who did not present for testing

- General feedback.

Method for collection and management of data

Data collection was done using two methods: A close-ended survey and social media analysis. 
The close-ended questionnaire (appendix 1) collected information related to factors contributing to non-presentation for testing. The survey was converted to a digital survey using Survey CTO. After selecting participants, randomly (using defined criteria), they were contacted by phone by trained data collectors. The data collectors informed the participants about the study, explained the objectives of the study and reassured respondents about the confidentiality of the process.

In addition to the survey, we collected social media data from Twitter on the sentiments around COVID-19 Testing in Nigeria. Using the Twitter Search API, recent tweets for Nigeria were gathered in nine days intervals over a month from April 16 through May 11 2020. We used the Twitter Search API's "geocode" parameter to retrieve the most recent tweets within Nigeria. The latitude of 9.881, longitude of 8.88, and radius of 394.571 miles were used. We used a set of covid-testing terms: "COVID", "testing", and "corona". To prepare the dataset for sentiment analysis, we removed all nonLatin characters from the tweets, and all links within the dataset. This process results in a dataset of 6,294 tweets from 3,909 users.

\section{Study participants}

A total of 566 participants had been invited for testing as of $16^{\text {th }}$ April 2020.

\section{Sample size and selection of participants}

Calculation of the sample was based on a simple random sampling for a finite population, using the formula:

$$
\text { Sample size }=\frac{\frac{z^{2} \times p(1-p)}{e^{2}}}{1+\left(\frac{z^{2} \times p(1-p)}{e^{2} N}\right)}
$$

Where $\mathrm{N}=$ population size, $\mathrm{e}=$ Margin of error (percentage in decimal form), $\mathrm{z}=\mathrm{z}$-score. $\mathrm{p}=$ expected prevalence. I.e.

- Population size: 566

- Acceptable margin of error: $5 \%$

- $\quad$ Confidence level: 95\% yielding $\mathrm{z}=1.96$

- $\quad$ Expected prevalence: 50\% to have higher sample size and because no single prevalence is measured in this study.

This yielded a total sample of 229 (by calculation) + 71 (security margin for non-response) $=300$. From the 566 invited par- ticipants, the 300 study participants were selected using a simple computerized random sampling in STATA 16.

\section{Data analysis}

Data from the closed-ended questionnaire were automatically downloaded from a secure server and transmitted real time by Survey CTO. High frequency checks were conducted, with variables like age, location etc. being compared with data in the existing database. Data management was conducted using STATA 16. Descriptive statistics were produced using proportions pertaining to selected variables. Further analysis looking for association, using odds ratios (OR) with 95\% confidence interval (CI) and including bivariate and multivariate unconditional logistic regression, was conducted using STATA 16. The main variables tested for associations were: outcome variable: present for testing (binary, 1 = yes and $0=$ no) against demographic and socioeconomic variables (age, gender, marital status, education level, location of residence, employment status) health status variables (pre-existing conditions, awareness about COVID-19 symptoms, source of health information), previous experience in healthcare facilities, satisfaction with NIMR service during COVID-19 (motivation to come for the testing, recommendation of service to others, challenges from form experience) mode of transport used and general feedback.

Each factor identified as significant in the bivariate analysis (in the form of an OR - with CI which is significantly different from 1), was integrated in a multivariate logistic regression model for analysis.

\section{Ethical considerations}

The study passed through the NIMR Institutional Review Board. The specific issues of ethical concern taken into account include:

- Informed consent: The voluntary and revocable agreement of an individual to participate in the study and based on an adequate understanding of its nature, purpose, and implications was required via verbal consent at the start of the questionnaire and at the end of the online registration form. The process will be used to ensure that the right and dignity of the human subject is protected as per ethics requirement.

- Privacy, confidentiality and data protection: Various precautions will be used to guarantee the confidentiality of information collected. Questionnaires and other documents holding respondent information will be secured in encrypted, private servers and protective measures will be estab- 
lished. Data cleaning and data entry will be performed by a statistician and data manager by using the case id without names' reference. The data collectors also signed confidentiality clauses.

- Anonymity: Measure will be taken to ensure that informants cannot be identified as the source of information expressed. This will be achieved by storing data without Personally Identifiable Information (PII).

- Data management and use: Data generated during and after the implementation of the study will be in joint custody of CoCreation HUB Limited and Nigeria Institute of Medical Research (NIMR). Access to and use of such data will comply with the existing approval mechanisms where the request is examined and authorization is guaranteed by NIMR and CcHUB leadership. Access and use for any other reasons will also be subject to leadership approval

\section{Results}

\section{Descriptive results}

Sample description

A total of 268 calls out of the targeted 300 were successfully made, reflecting a response rate of $89 \%$. Of the $268,78 \%(n=210)$ gave consent to be interviewed. The respondents were pre-classified into two groups: (i) those who presented themselves for testing or (ii) those who did not present themselves for testing. The analysis of this research is set upon comparison of the two groups.

\section{Demographic and socioeconomic information}

\section{Gender}

Majority $(70 \%, n=147)$ of the overall respondents were male and they were also the majority across both groups. $65 \%(n=73)$ of those who presented for testing were male and $(76 \%, n=74)$ of those who did not show up for testing were also male. Chi square test to check for association between gender and presenting oneself for testing revealed no association (Chsq=2.65) as shown in table 2 below.

\begin{tabular}{|l|c|c|c|c|c|c|c|c|c|}
\hline \multirow{2}{*}{ Gender } & \multicolumn{2}{|c|}{ Presented self for testing } & \multicolumn{2}{c|}{ Did not present self for testing } & \multicolumn{3}{c|}{ Total } \\
\cline { 2 - 10 } & Base & $\mathbf{N}$ & $\mathbf{\%}$ & Base & $\mathbf{n}$ & $\mathbf{\%}$ & Base & n & \% \\
\hline Male & 112 & 73 & $65 \%$ & 98 & 74 & $76 \%$ & 210 & 147 & $70 \%$ \\
\hline Female & 112 & 39 & $35 \%$ & 98 & 24 & $24 \%$ & 210 & 63 & $30 \%$ \\
\hline
\end{tabular}

Table 2: Gender description of respondents.

Age

The age of the respondents ranged from 19 to 87 with a mean and median age being 37 and 35 years respectively. More than half $(67 \%, n=141)$ of the respondents were aged between 20 to 39 years, with those between 30 and 39 years forming the larger portion $(40 \%, n=85)$ of the demographic. In addition, the 30 to 39 age group is the same demographic for those who turned up for testing and those who did not (38\%, $\mathrm{n}=42$ and $44 \%, \mathrm{n}=43$ respectively). The average age of those who presented themselves for testing is 39 (10), and 34 (10) for those who did not present for testing. A t-test conducted to compare the average age difference between the two groups revealed that those who presented themselves for testing are slightly older than those who did not, at $5 \%$ significance level $(\mathrm{t}=-3.2 .186, \mathrm{df}=208)$. This indicates that the higher show up rates are skewed towards the younger age groups as shown in figure 1 below.
Respondent Age Groups Distribution

aresented self for testing $\quad$ Did not present self for testing

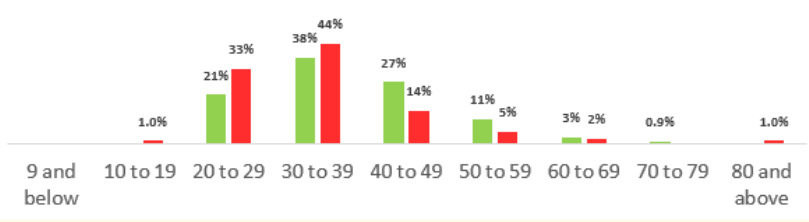

Figure 1: Age group distribution of respondents.

\section{Marital status}

More than half $(54 \%, n=113)$ of the respondents were married, while majority $(45 \%, n=95)$ of the rest were never married. 
The remaining $1 \%$ were divorced. The majority $(59 \%, \mathrm{n}=66)$ of those who turned up for testing were married, while the majority $(52 \%, \mathrm{n}=51)$ of those who did not, have never been married, as shown in the table below. However, chi square tests for association showed marital status and turned up for testing are not associated (chisq=4.78).

\begin{tabular}{|l|c|c|c|c|c|c|c|c|c|}
\hline \multirow{2}{*}{ Marital Status } & \multicolumn{3}{|c|}{$\begin{array}{c}\text { Presented self for } \\
\text { testing }\end{array}$} & \multicolumn{3}{c|}{$\begin{array}{c}\text { Did not present } \\
\text { self for testing }\end{array}$} & \multicolumn{4}{c|}{ Total } \\
\cline { 2 - 11 } & Base & $\mathbf{n}$ & \% & Base & n & \% & Base & n & \% \\
\hline Married & 112 & 66 & $59 \%$ & 98 & 47 & $48 \%$ & 210 & 113 & $54 \%$ \\
\hline Divorced & 112 & 2 & $2 \%$ & 98 & 0 & $0 \%$ & 210 & 2 & $1 \%$ \\
\hline Widowed & 112 & 0 & $0.0 \%$ & 98 & 0 & $0.0 \%$ & 210 & 0 & $0.0 \%$ \\
\hline Never married & 112 & 44 & $39 \%$ & 98 & 51 & $52 \%$ & 210 & 95 & $45 \%$ \\
\hline
\end{tabular}

Table 3: Marital status.

\section{Education level}

When asked about the highest level of education, $60 \%(n=125)$ reported that they have completed a bachelor's degree. Similarly, more than half $(58 \%, n=65)$ of those who presented for testing and majority $(61 \%, \mathrm{n}=27)$ of those who did not present themselves for testing have a bachelor's degree. These results show that more than $90 \%$ of the applicants who filled the online form have at least a bachelor's degree, as shown in the table 4 below.

\begin{tabular}{|l|c|c|c|c|c|c|c|c|c|}
\hline \multirow{2}{*}{ Education level } & \multicolumn{3}{|c|}{$\begin{array}{c}\text { Presented self for } \\
\text { testing }\end{array}$} & $\begin{array}{c}\text { Did not present self } \\
\text { for testing }\end{array}$ & \multicolumn{3}{c|}{ Total } \\
\cline { 2 - 12 } & Base & $\mathbf{n}$ & $\mathbf{\%}$ & Base & $\mathbf{n}$ & $\mathbf{\%}$ & Base & $\mathbf{n}$ & $\%$ \\
\hline None. kindergarten & 112 & 0 & $0.0 \%$ & 98 & 0 & $0.0 \%$ & 210 & 0 & $0.0 \%$ \\
\hline Primary & 112 & 0 & $0.0 \%$ & 98 & 0 & $0.0 \%$ & 210 & 0 & $0.0 \%$ \\
\hline secondary & 112 & 3 & $3 \%$ & 98 & 4 & $4 \%$ & 210 & 7 & $3 \%$ \\
\hline Vocational/technical education & 112 & 5 & $4 \%$ & 98 & 8 & $8 \%$ & 210 & 13 & $6 \%$ \\
\hline Bachelor's degree & 112 & 65 & $58 \%$ & 98 & 60 & $61 \%$ & 210 & 125 & $60 \%$ \\
\hline Postgraduate degree & 112 & 39 & $35 \%$ & 98 & 26 & $27 \%$ & 210 & 65 & $31 \%$ \\
\hline
\end{tabular}

Table 4: Education level.

\section{State of residence}

Over two-thirds $(89 \%, \mathrm{n}=186)$ of the respondents reside in Lagos State. The rest mentioned that they reside in Ogun $(4 \%, \mathrm{n}=$ 9), Oyo ( $4 \%, \mathrm{n}=8$ ), Kano, Kebbi, Edo, Anambra, Kwara and Akwa Ibom states $3 \%(n=7)$ as shown in the table below. Chi square tests of association showed that there is a statistically significant association between the state of residence and presenting one self for testing or not at $5 \%$ significance level (chisq=4.3). The majority of those who turned up for testing and also majority of those who did not reside in Lagos (93\%, $\mathrm{n}=112$ and 84\%, $\mathrm{n}=82$ respectively).

\begin{tabular}{|c|c|c|c|c|c|c|c|c|c|}
\hline \multirow{2}{*}{ State of residence } & \multicolumn{3}{|c|}{ Presented self for testing } & \multicolumn{3}{|c|}{ Did not present self for testing } & \multicolumn{3}{|c|}{ Total } \\
\hline & Base & $\mathbf{n}$ & $\%$ & Base & $\mathbf{n}$ & $\%$ & Base & $\mathbf{n}$ & $\%$ \\
\hline Lagos & 112 & 104 & $93 \%$ & 98 & 82 & $84 \%$ & 210 & 186 & $89 \%$ \\
\hline Ogun & 112 & 4 & $4 \%$ & 98 & 5 & $5 \%$ & 210 & 9 & $4 \%$ \\
\hline Kano & 112 & 1 & $1 \%$ & 98 & 1 & $1 \%$ & 210 & 2 & $1 \%$ \\
\hline Oyo & 112 & 1 & $1 \%$ & 98 & 7 & $7 \%$ & 210 & 8 & $4 \%$ \\
\hline Kebbi & 112 & 0 & $0.0 \%$ & 98 & 1 & $1.0 \%$ & 210 & 1 & $0.5 \%$ \\
\hline Edo & 112 & 1 & $0.9 \%$ & 98 & 0 & $0.0 \%$ & 210 & 1 & $0.5 \%$ \\
\hline Anambra & 112 & 1 & $0.9 \%$ & 98 & 0 & $0.0 \%$ & 210 & 1 & $0.5 \%$ \\
\hline Kwara & 112 & 0 & $0.0 \%$ & 98 & 1 & $1.0 \%$ & 210 & 1 & $0.5 \%$ \\
\hline Akwa_Ibom & 112 & 0 & $0.0 \%$ & 98 & 1 & $1.0 \%$ & 210 & 1 & $0.5 \%$ \\
\hline
\end{tabular}

Table 5: State of residence. 


\section{Employment category}

A total of 156 (74\%) respondents reported that they are formally employed. Similar proportions are seen among people who presented themselves for testing $(78 \%, \mathrm{n}=87)$ and those who did not $(70 \%, n=69)$ as shown in the figure 2 below.

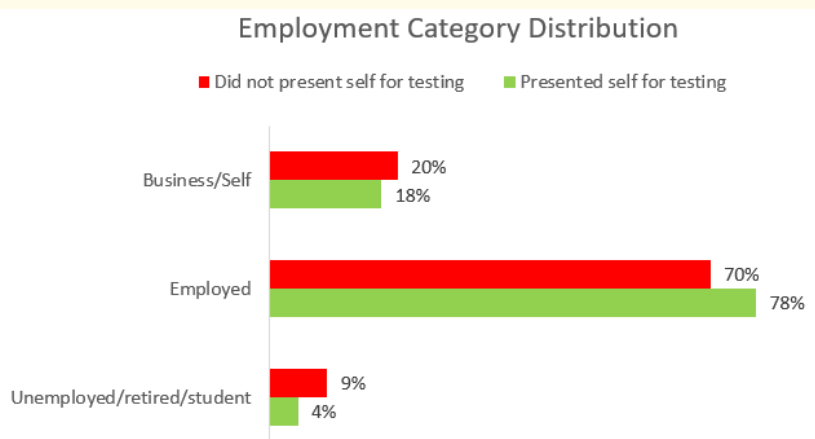

Figure 2: Employment category.

\section{Health information}

Respondents were required to provide information about preexisting health conditions they had, their knowledge of COVID-19 symptoms and their source of health information.

\section{Preexisting health conditions}

We asked the respondents if they had any preexisting health conditions, specifically pregnancy for female respondents, heart diseases, asthma or chronic lung diseases or any form of disability. Our analysis showed that on average, respondents had no preexisting conditions. $20.5 \%(n=43)$ of all respondents had between 1 and 3 preexisting health conditions, about a quarter of which $(27 \%, n=31)$ presented themselves for testing. Majority $(88 \%, n$ $=86$ ) of those who did not turn up to be tested had no preexisting health conditions as shown in the table below. The preexisting health conditions that the respondents mentioned included heart disease $(7 \%, \mathrm{n}=15)$, asthma and other chronic diseases $(9 \%, \mathrm{n}=$ 18 ) and goitrogenic disease/ulcers $(3 \%, n=6)$.

\begin{tabular}{|c|c|c|c|c|c|c|c|c|c|c|}
\hline \multirow[t]{2}{*}{ Question } & \multirow[t]{2}{*}{ Option } & \multicolumn{3}{|c|}{$\begin{array}{c}\text { Presented self for } \\
\text { testing }\end{array}$} & \multicolumn{3}{|c|}{$\begin{array}{c}\text { Did not present self } \\
\text { for testing }\end{array}$} & \multicolumn{3}{|c|}{ Total } \\
\hline & & Base & $\mathbf{n}$ & $\%$ & Base & $\mathbf{N}$ & $\%$ & Base & $\mathbf{n}$ & $\%$ \\
\hline \multicolumn{2}{|c|}{ Average/median number of preexisting conditions } & 112 & & 0 & 98 & & 0 & 210 & & 0 \\
\hline \multirow{4}{*}{$\begin{array}{l}\text { Number of preexisting } \\
\text { conditions one has }\end{array}$} & 0 & 112 & 81 & $72.3 \%$ & 98 & 86 & $87.8 \%$ & 210 & 167 & $79.5 \%$ \\
\hline & 1 & 112 & 27 & $24.1 \%$ & 98 & 10 & $10.2 \%$ & 210 & 37 & $17.6 \%$ \\
\hline & 2 & 112 & 4 & $3.6 \%$ & 98 & 1 & $1.0 \%$ & 210 & 5 & $2.4 \%$ \\
\hline & 3 & 112 & 0 & $0.0 \%$ & 98 & 1 & $1.0 \%$ & 210 & 1 & $0.5 \%$ \\
\hline
\end{tabular}

Table 6: Health preexisting conditions.

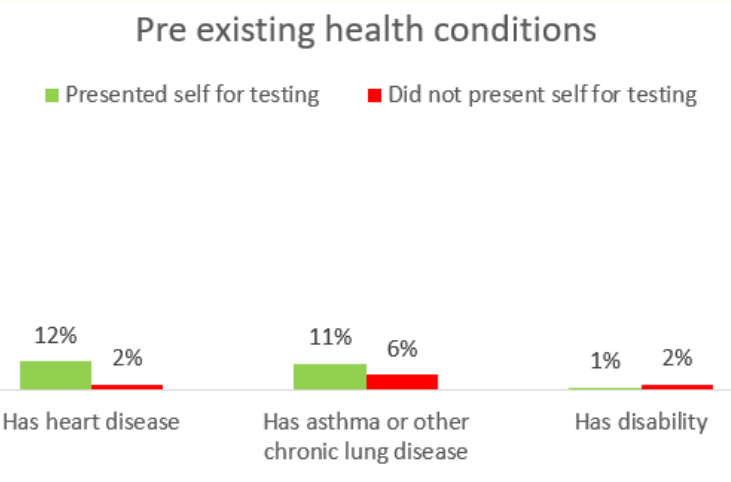

Figure 3: Pre-existing health conditions.
Knowledge of COVID-19 symptoms

We also asked the respondents if they knew any symptoms of COVID-19. Almost all $(99 \%, n=208)$, answered to the affirmative. This was observed in both groups of respondents.

When asked to specify the symptoms they are aware of, respondents were found to know on average 4 symptoms, out of the 11 listed by CDC [7]. This was the average number of symptoms for respondents who had presented themselves for testing and those who did not. However, $42 \%(n=89)$ of the respondents knew more than half of the 11 symptoms listed by CDC. The top 4 most frequently mentioned symptoms were cough $(92 \%, \mathrm{n}=193)$, fever $(90 \%, n=189)$, shortness of breath $(79 \%, n=166)$ and sore throat $(60 \%, n=125)$. Respondents reported limited knowledge about some of the symptoms such as loss of taste $(1.9 \%, n=6)$, loss of 
smell $(2.4 \%, \mathrm{n}=5)$ and back and abdominal pains $(2.9 \%, \mathrm{n}=6)$ as shown in figure 4 below.

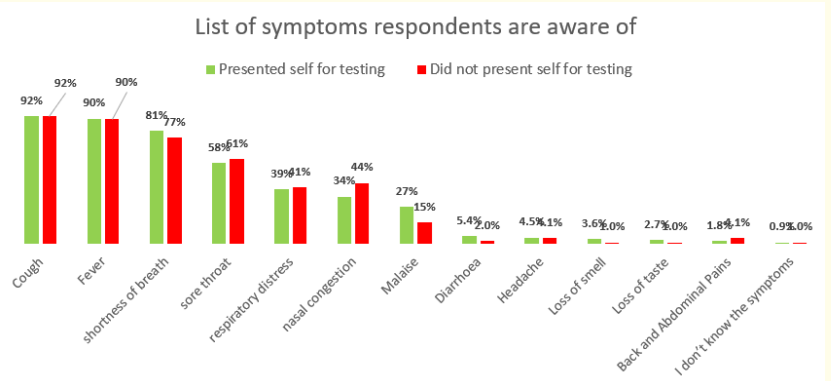

Figure 4: List of symptoms respondents know.

Source of health information and use of social media

Respondents were asked about their source of information on health and healthcare. Some of the most mentioned sources in- clude social media $(79 \%, \mathrm{n}=165)$, radio and television $(42 \%, \mathrm{n}=$ $89)$ and Ministry of Health website $(29 \%, n=61)$. Additionally, in response to frequency of social media use, more than two thirds $(88 \%, \mathrm{n}=184)$ of all respondents stated that they used it three or more times in a day as shown in table 7.

Twitter data sentiment analysis results

This analysis provides a way to understand the attitudes and opinions expressed in texts (tweets), and to understand if and how a narrative changes over time. The algorithm used (bing lexicon) categorizes words in a binary fashion (into positive and negative) categories. During the period of April 16 to May 11 2020, the sentiments expressed around COVID-19 Testing in Nigeria were alternating, however, negative attitudes kept dominating the discussion, and the highest peak was observed on April, $27^{\text {th }} 2020$, when His Excellency Muhammadu Buhari was addressing Nigerians on the COVID-19 pandemic and response activities (Figure 5).

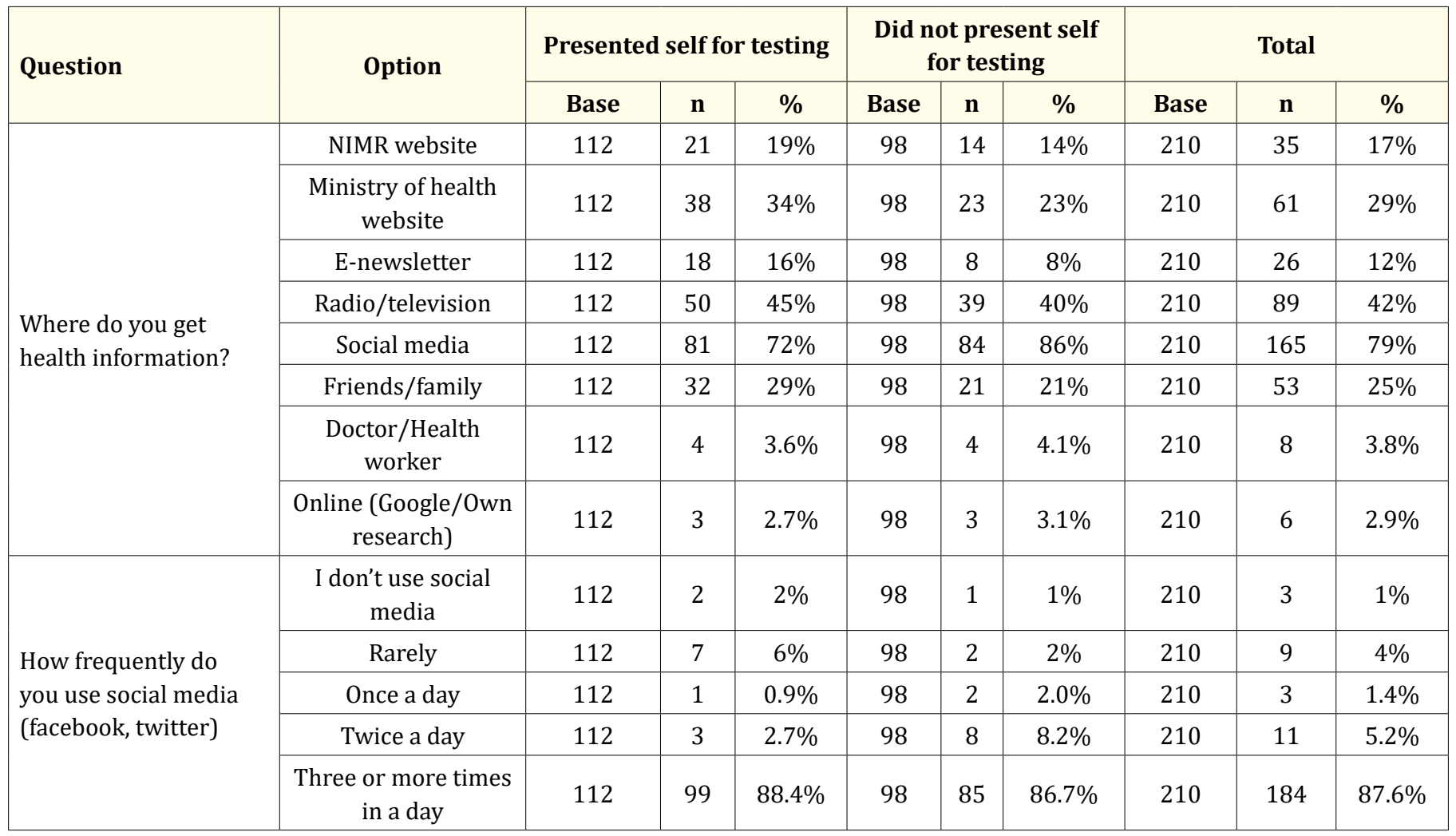

Table 7: Source of health information. 


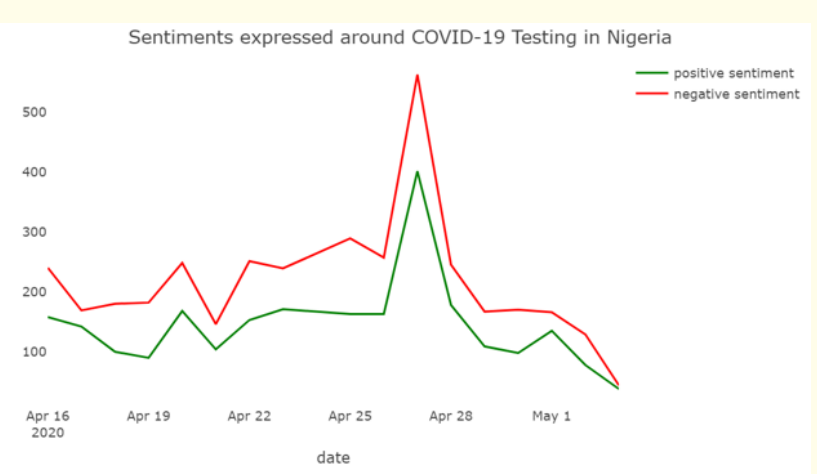

Figure 5: Twitter sentiments around COVID testing in Nigeria.
Most tweets reflected negative sentiments, with virus $(n=495)$ as the top-ranked term contributing to sentiments. Tweet categories that reflected more than 100 times negative sentiments were: virus, isolation, symptoms, deaths, and infected. Tweets mentioning free, cure, rapid, support, safe, and ready contributed a lot to positive sentiments (Figure 6).

"You think brother taju that lives in the same compound with you doesn't have covid-19, shior. Someone that is already walking with coronavirus. 70 new cases in lagos. So many townsfolk are still walking with the virus. More testing needs to be done. Don't trust

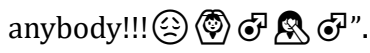

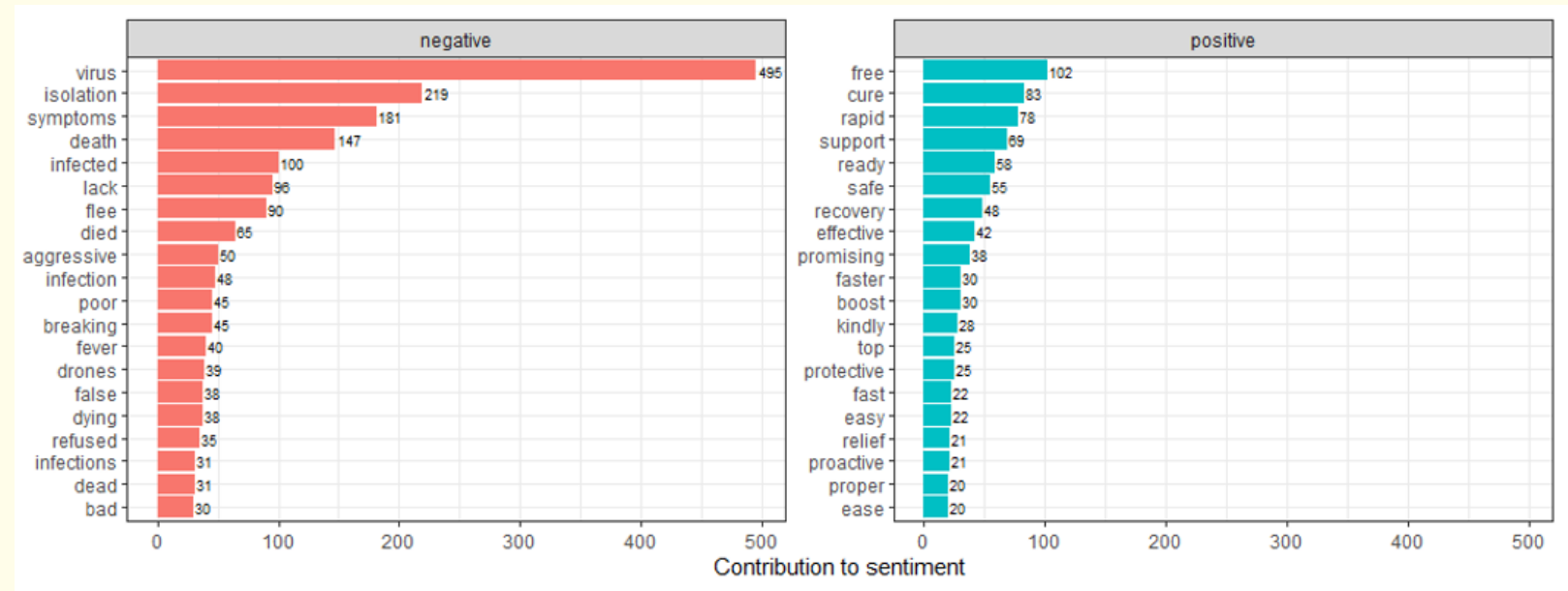

Figure 6: Phrases in sentiments on COVID-19 testing in Nigeria.

\section{[heytwofficial]}

"You Can't Detect COVID-19 Without Testing - NCDC To Unwilling State Govts - https://t.co/bRbSIRJiiH Director General of the NCDC, Dr. Chike Ihekweazu has reiterated that testing to detect COVID-19 is a no-brainer and is something that has to be done to detect the scale of the... https://t.co/HDR6cWz0d9".

\section{[AledehLive]}

\section{Previous experience with healthcare system}

Sentiments varied for respondents who rated various aspects (communication, waiting time, treatment received, cleanliness and overall satisfaction) of the experience received from healthcare facilities they routinely utilize. Communication, waiting time, cleanliness and overall satisfaction received more positive than negative ratings by respondents $(46 \%, \mathrm{n}=97$ for communication, $49 \%, \mathrm{n}$ $=103$ for waiting time, $61 \%, \mathrm{n}=128$ for cleanliness and $57 \%, \mathrm{n}=$ 116 for overall satisfaction) as shown in the figure below.
Ratings based on previous heath facility visit for all respondents

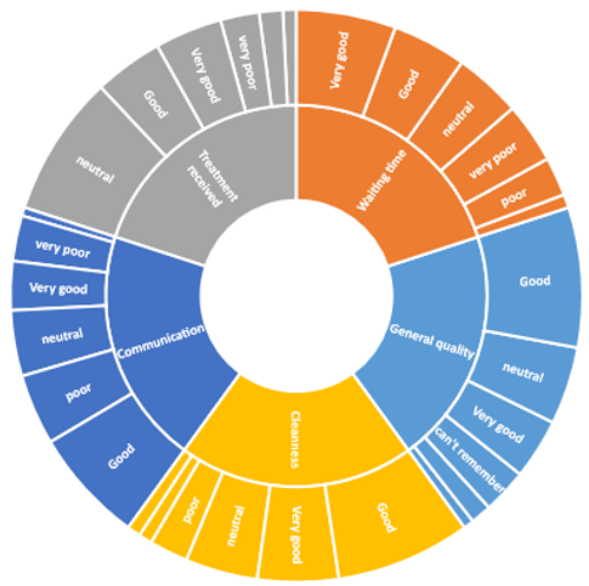

Figure 7: Ratings based on previous health facility visit. 
Experience with NIMR service during COVID-19

All the respondents that spoke about their experience with NIMR were drawn from a pool of citizens who at least filled the online form to apply for the free COVID-19 testing being provided by NIMR. We therefore sought to know their experience with the service NIMR provided across the various stages of the testing process.

\section{Pre-testing experience}

Majority $(95 \%, n=199)$ of the respondents stated that they were satisfied with the process of filling the online form. This was the case for those who presented themselves for testing (94\%, n communication after filling the form, the majority $(89 \%, n=186)$ reported that they received clear instructions in the communication, however, most $(57 \%, \mathrm{n}=120)$ communication was received by email only. The chi square tests of association show that means of communication after online form submission is significantly associated with whether a person showed up for testing. This is particularly seen when $12 \%(n=12)$ of those who did not present themselves for testing reported that they did not receive any communication after filling the form, and $53 \%(n=52)$ of the same group stated that they only received emails as shown in the table 8 below.

\begin{tabular}{|c|c|c|c|c|c|c|c|c|c|c|}
\hline \multirow[t]{2}{*}{ Question } & \multirow[t]{2}{*}{ Option } & \multicolumn{3}{|c|}{ Presented self for testing } & \multicolumn{3}{|c|}{$\begin{array}{c}\text { Did not present self } \\
\text { for testing }\end{array}$} & \multicolumn{3}{|c|}{ Total } \\
\hline & & Base & $\mathbf{n}$ & $\%$ & Base & $\mathbf{n}$ & $\%$ & Base & $\mathbf{n}$ & $\%$ \\
\hline \multirow{2}{*}{$\begin{array}{l}\text { Were you satisfied with } \\
\text { the process of filling } \\
\text { the online form? }\end{array}$} & Yes & 112 & 105 & $94 \%$ & 98 & 94 & $96 \%$ & 210 & 199 & $95 \%$ \\
\hline & No & 112 & 7 & $6 \%$ & 98 & 4 & $4 \%$ & 210 & 11 & $5 \%$ \\
\hline \multirow{4}{*}{$\begin{array}{l}\text { How were you com- } \\
\text { municated with after } \\
\text { filling the form? }\end{array}$} & Email & 112 & 68 & $61 \%$ & 98 & 52 & $53 \%$ & 210 & 120 & $57 \%$ \\
\hline & Phone call & 112 & 14 & $13 \%$ & 98 & 27 & $28 \%$ & 210 & 41 & $20 \%$ \\
\hline & Email and phone call & 112 & 22 & $20 \%$ & 98 & 7 & $7 \%$ & 210 & 29 & $14 \%$ \\
\hline & None & 112 & 8 & $7 \%$ & 98 & 12 & $12 \%$ & 210 & 20 & $10 \%$ \\
\hline \multirow{2}{*}{$\begin{array}{l}\text { Were you given clear } \\
\text { instructions? }\end{array}$} & Yes & 112 & 101 & $90 \%$ & 98 & 85 & $87 \%$ & 210 & 186 & $89 \%$ \\
\hline & No & 112 & 11 & $10 \%$ & 98 & 13 & $13 \%$ & 210 & 24 & $11 \%$ \\
\hline
\end{tabular}

Table 8: Pre testing experience.

\section{Motivation for testing}

We inquired about motivating factors for those who showed up for testing at the testing center. Majority $(41 \%, n=45)$ reported that they experienced some of the symptoms, while the other top 2 reasons included knowledge of their status $(10 \%, n=22)$ and history of contact with friends or relatives with COVID-19 symptoms $(14 \%, \mathrm{n}=15)$ as shown in the figure below.

\section{Motivation to present one self for testing}

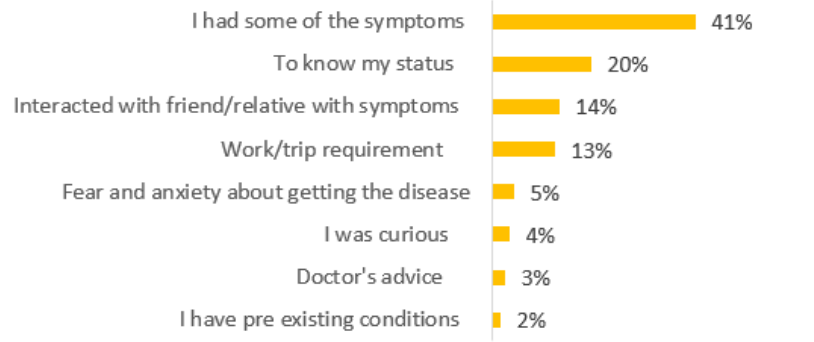

\section{Post testing experience and recommendation}

When asked if they would recommend NIMR services to anyone, more than $90 \%$ ( $n=102$ ) of those who presented themselves for testing stated that they are either likely or very likely to do so. We sought to find out if those who presented themselves for testing experienced any challenges or issues. Half of the respondents ( $\mathrm{n}$ = 55) mentioned they had no challenges or issues, while $17 \%$ ( $\mathrm{n}$ $=19)$ and $12 \%(n=13)$ of the respondents highlighted poor time management and inadequate testing capacity as areas for improvement respectively as shown in the table below (Table 9).

One of the respondents, for example, mentioned that "Enough personnel and equipment should be provided at the site to reduce waiting times for people who are to be tested".

Another one also highlighted that "Increase the testing capacity and the awareness around the availability of the testing to encourage more people to come for it".

Figure 8: Motivation for testing 


\begin{tabular}{|l|c|c|c|}
\hline \multirow{2}{*}{$\begin{array}{l}\text { Issues/Challenges from } \\
\text { COVID-19 testing experience }\end{array}$} & \multicolumn{3}{|c|}{ Presented self for testing } \\
\cline { 2 - 4 } & Base & $\mathbf{n}$ & $\mathbf{\%}$ \\
\hline None & 111 & 55 & $50 \%$ \\
\hline Poor time management & 111 & 19 & $17 \%$ \\
\hline Transport challenges & 111 & 2 & $2 \%$ \\
\hline Inadequate testing capacity & 111 & 13 & $12 \%$ \\
\hline No/late feedback & 111 & 8 & $7 \%$ \\
\hline Poor site management & 111 & 4 & $4 \%$ \\
\hline Incorrect personal details & 111 & 5 & $5 \%$ \\
\hline Repetitive questions & 111 & 1 & $1 \%$ \\
\hline Poor communication & 111 & 4 & $4 \%$ \\
\hline
\end{tabular}

Table 9: Post testing experience.

Mobility to testing center

We asked those who presented themselves for testing what mode of transport they used to reach the testing facility, and asked those who were yet to turn up for testing how they intended to get to the testing facility. Majority $(91 \%, \mathrm{n}=102)$ of those who presented themselves to the testing facility did so by car. On the other hand, the majority $(60 \%, n=59)$ of those who were yet to present themselves mentioned that they would use cars. However, $23 \%(n=23)$ of those yet to show up highlighted that they do not have a means of getting to the testing center. All this is shown in the figure below.

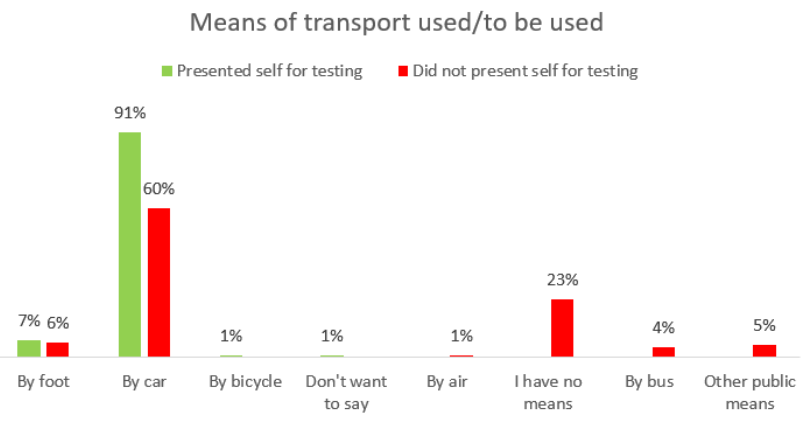

Figure 9: Modes of transport.

Finally, we asked all respondents if they had any comments or feedback to share. Based on the sentiments from each feedback or comment, they were categorized into three groups, namely: positive feedback, neutral feedback and improvement feedback. Most $(54 \%, \mathrm{n}=104)$ mentioned that they had no feedback to share, $31 \%$ ( $n=59$ ) of the remaining responders provided feedback on areas for improvement. One of particular interest is feedback from both groups that they experienced delays in getting results and feedback from the testing center, as shown in the table below.

\begin{tabular}{|c|c|c|c|c|c|c|c|c|c|}
\hline \multirow{2}{*}{ Feedback } & \multicolumn{3}{|c|}{ Presented self for testing } & \multicolumn{3}{|c|}{ Did not present self for testing } & \multicolumn{3}{|c|}{ Total } \\
\hline & Base & $\mathbf{n}$ & $\%$ & Base & $\mathbf{n}$ & $\%$ & Base & $\mathbf{n}$ & $\%$ \\
\hline Positive - wants to be tested & 98 & 0 & $0 \%$ & 93 & 4 & $4 \%$ & 191 & 4 & $2 \%$ \\
\hline Positive - good professionalism & 98 & 3 & $3 \%$ & 93 & 2 & $2 \%$ & 191 & 5 & $3 \%$ \\
\hline Neutral- general & 98 & 0 & $0 \%$ & 93 & 1 & $1 \%$ & 191 & 1 & $1 \%$ \\
\hline Improvement - transport challenge & 98 & 1 & $1 \%$ & 93 & 4 & $4 \%$ & 191 & 5 & $3 \%$ \\
\hline Improvement - poor time management & 98 & 3 & $3 \%$ & 93 & 1 & $1 \%$ & 191 & 4 & $2 \%$ \\
\hline Improvement - form issues/improvement & 98 & 4 & $4 \%$ & 93 & 2 & $2 \%$ & 191 & 6 & $3 \%$ \\
\hline Improvement - fears about privacy & 98 & 0 & $0 \%$ & 93 & 1 & $1 \%$ & 191 & 1 & $1 \%$ \\
\hline Improvement - closer test centers & 98 & 4 & $4 \%$ & 93 & 7 & $8 \%$ & 191 & 11 & $6 \%$ \\
\hline
\end{tabular}

Table 10: Feedback. 
Two respondents who turned up for testing mentioned: "I Informed others at work to register. They all didn't get any feedback" And

"I was dissatisfied by lack of feedback on the test status".

Others who were yet to present mentioned that: "I didn't get a response even after filling the form".

And

"I should have been contacted when I didn't make the appointment".

\section{Inferential results}

The main variables tested using inferential analysis for associations were: outcome variable: present for testing (binary, 1=yes and $0=$ no) against demographic and socioeconomic variables (age, gender, marital status, education level, location of residence, employment status) health status variables (pre-existing conditions, awareness about COVID-19 symptoms, source of health information), previous experience in healthcare facilities, satisfaction with NIMR service during COVID-19 (motivation to come for the testing, recommendation of service to others, challenges from form experience) mode of transport used and general feedback. All tests were done using 5\% level of significance, and multivariate models were fitted for all variables that were significant in bivariate analysis.

\section{Demographic and socioeconomic information}

Bivariate logistic regression fitted on demographic and socioeconomic information against the dependent variable showed that age, age groups, state of residence, type of LGA one resides were significantly associated with turning up for testing at 5\% significance level. Specifically, as age increases, the odds of turning up for testing increased with an OR (CI 95\%) of 1.047 [1.017,1.079]. Those who fall in the age groups 40-49 and 50-59 were more likely to turn up for testing respectively an OR (CI 95\%) of 2.857 [1.251,6.527] and 3.2 [0.993,10.309]. On the other hand, those who reside out of Lagos were $61 \%[0.161,0.966]$ less likely to turn up for testing, compared to those invited and living within Lagos. Compared to those who reside in semi urban LGAs, those who live in rural LGAs were $70 \%[0.121,0.717]$ less likely to turn up for testing.

When a multivariate logistic regression model was fitted only age and those who fall within the age group 30-39 were more likely to turn up for testing with OR (CI 95\%) of 1.18 [1.018,1.367] and $0.188[0.042,0.835]$ respectively. As shown on appendix 2 .

\section{Health information}

From respondent's health information, patients without preexisting heart conditions and those who identified malaise as a COVID-19 symptom were significantly less likely and more likely to turn up for testing with OR (CI 95\%) of 0.159 [0.035,0.722] and $2.024[1.015,4.039]$ respectively. However, upon fitting the multivariate logistic regression model, there was insufficient evidence to support association of the variables with presenting oneself for testing as shown on Appendix 2.

\section{Previous experience with healthcare system}

Bivariate logistic regression fitted on ratings from previous experience with healthcare system showed that people who rated communication as neutral, those who couldn't remember how they were treated and those who rated general hospital quality were less likely to present themselves for testing an OR (CI 95\%) 0.358 [0.134,0.956], 0.095 [0.011,0.85], and 0.394 [0.159,0.977] respectively However, those who rated waiting time as good were more likely to turn up for testing with an OR (CI 95\%) 5.253 $[1.406,19.62]$. Once a multivariate logistic regression model was fitted, the variables were no longer associated with tuning up for testing as shown on Appendix 2.

\section{Experience with NIMR service during COVID 19}

Responses to various stages of interacting with NIMR service during COVID19 showed that compared to those who only received email communication, those who received phone calls only were less likely to turn up for testing with an OF (CI 95\%) of 0.397 $[0.189,0.831]$. This was confirmed by the multivariate logistic regression with an OR (CI 95\%) of 0.241 [0.94,13.519] as shown in Appendix 2.

\section{Discussion}

Existing literature shows that the need for health-care services to improve or maintain health are major determinants of healthcare utilization. The World Health Organization states that health is determined by a person's individual characteristics and behaviors, physical environment, and socioeconomic environment [8].

A person's characteristics such as age, gender, disability status, employment status and other determinants of health such as education have been found to correlate with healthier populations [9]. Findings from Thomas-Henkel and Schulman [10], show that people who have unmet social needs are more likely to not show up for medical appointments. 
Generally, more men than women registered for testing, and we also see more men turning up for testing in the study. This is contrary to some studies conducted in USA [11] Hong Kong [12] (2002), which have shown that women make more primary care visits and are more likely to seek diagnostic services, screening uptake (cancer and HIV), diet and nutrition counseling. It, however, agrees with research conducted in South Africa on TB patients [13].

Age distribution of those turning up for COVID-19 testing is skewed towards the younger age group. Specifically, those aged 30-39. Those from older populations, (above 50 years old), have the lowest turn up rates. This agrees with previous research conducted in the USA [14] Burkina Faso [15] and Nigeria [16] that show that age is an important factor in care seeking behaviour. Specifically, increased patient delay to seeking treatment was associated with older age. This is of concern since the CDC stated that the older population has a high risk of being infected with $\mathrm{CO}$ VID-19 [7].

Although not significant, there was a higher percentage turn up from married participants compared to unmarried participants. Preliminary analysis of data from the registration form had shown that these married participants were turning up with their spouses. It is of interest to note, given that no literature has actually shown that marital status influences in one way or another care seeking behaviour.

The overall result for the level of education of participants shows that people coming for testing are educated. Previous studies conducted on the Zlka outbreak in Brazil showed that the level of education only influences care seeking behaviour when it is paired with knowledge of the disease. Those highly educated, were correlated with more knowledge of the disease and therefore were more likely to seek healthcare services [17]. However, this was not comparable in our study because the majority of the respondents were degree (bachelor and postgraduate) holders and there was insufficient evidence to support any association between education level and turning up for testing.

People who applied for testing from outside Lagos state were less likely to turn up for testing, as revealed by the chi-square test. Compared to those who reside in semi urban LGAs, those who live in rural LGAs were less likely to turn up for testing. This agrees to what some research studies have shown. A study on care seeking behavior during SARS indicates that hard to reach areas can imply geographic remoteness or social isolation where people live on the outskirts of mainstream society. This, they report, could also pose a barrier for patient treatment seeking measure [18]. The possible reasons for this are reflected in the means of transportation for coming for testing, though most of those residing outside Lagos showed interest in care seeking by stating that they really wanted to know their status. With increased concern about COVID-19 and limited access to testing centers, it is likely that those from outside Lagos and rural LGAs are not in a position to seize the opportunity to utilize the free testing.

Our study saw a higher percentage turn up from employed participants. This could be attributed to a motivation for showing up for testing - some employed participants needed test results for work. It also suggests employed people are more health-conscious because health is usually a requirement for maintaining employment, as seen in previous research $[19,20]$.

Heart disease, asthma or other chronic lung diseases, and diabetes have been reported to be a risk factor for severity of COVID-19 [21-23]. The higher percentage turn up from participants with these pre-existing health conditions suggests understanding of the COVID-19 health information being passed across by health organizations. This is confirmed by the lower percentage turn up from participants with diseases that have no relationship with the severity of COVID-19 (blindness, deafness, enlarged prostate, gastrogenic disease/ulcers, sinusitis, arthritis/bone conditions and eye issues). The higher percentage turn up from participants with pre-existing health conditions, compared to lower percentage turn up from participants with no pre-existing health conditions, confirms pre-existing health conditions influences healthcare seekers behavior. A study on vulnerability in an Influenza pandemic by Sullivan and Bourgoin [18] emphasizes that having some pre-existing conditions such as diabetes or conditions that predispose people to infectious diseases are linked to health seeking behavior, as well as compliance.

Symptoms reported by the participants are similar to those reported by CDC and WHO. However, the limited knowledge of some of the symptoms such as loss of taste, loss of smell, and back and abdominal pain, suggests reiteration has an effect on the people's knowledge of the COVID-19 disease. This is confirmed by the fact 
that symptoms that are usually reiterated by health organisations and the internet in general (cough, fever and shortness of breath) were the most known.

Participants who get health information from verified sources like the NIMR website, Ministry of Health website, and radio or television had a higher percentage of participants that turned up for testing than those who did not turn up and got information from the same sources. The reverse was the case for the category of participants that get health information from social media and online sources (google/own research). Both social media and online sources (google/own research) are crowded with false information of COVID-19 and its seriousness. In the early stages of the free testing provided by CcHUB, LifeBank and NIMR, there were numerous conspiracies about the COVID-19 pandemic. Social media and online sources contributed to the transmission of such conspiracies and people expressed negative sentiments around COVID-19 Testing through the twitter discussion. This suggests the low turn up may have been due to false information and not enough reiteration of the seriousness of COVID-19.

Participants with previous negative experiences with the cleanliness of healthcare systems had a lower percentage turn up, while those with positive previous experience had a higher percentage turn up. Hygiene is an important factor in COVID-19 infection/ transmission. The results therefore suggest participants understand the role hygiene plays in infection/transmission of COVID-19, and may have decided not to turn up for testing for fear of the cleanliness of the testing centre. It also confirms participants understand the health information on COVID-19 being disseminated. The higher percentage turn up in the category of participants who had positive previous experiences with the general quality of healthcare services, correlates with previous studies conducted in Nigeria and Uganda [24,25], which show that quality of healthcare service greatly influences healthcare seeking behavior.

Means of communication after filling the form had a significant association with the turn up of patients for testing. This is confirmed by the higher percentage of participants who turned up for the test than those who did not, in the category of those that received two means of communication, and the category that received an email only. The reverse was the case for the category that did not receive any communication and the category that received a phone call only. This correlates with previous studies [26,27] that suggest communication is a major influence on people's desire to seek healthcare.

The results from transport challenges and closer test centres for general feedback shows that location and mobility had an influence on the turn up of participants for testing. It is interesting to note that no one mentioned the service being free as a motivation for showing up for testing.

There were several limitations to this study, including implementation time. The COVID-19 pandemic situation is changing rapidly, globally. This means that the research methodology had to be adopted and implemented quickly. Also, given the safety measures surrounding COVID-19 including social distancing, robust qualitative data collection methods, particularly focus group discussions, could not be implemented. This would have provided more insight into the study. Another limitation was the use of calls as a data collection method. This resulted in the exclusion of people with no access to mobile phones. There is a risk that the sample does not include many people who are at the very bottom of the pyramid.

\section{Conclusion}

In conclusion, the significant differences in showing up for testing were found across gender, age differences, education levels, state of residence and employment status. The source of health information, having pre-existing health conditions and the experience with healthcare providers also played a crucial role in those that turned up for testing.

Our findings confirm the outcomes of several other studies. Age distribution of the people who turned up for COVID-19 testing is skewed towards the younger age group; people with 50 years old and above have the lowest turn up rates [14-16]. The level of education of participants shows that people coming for testing are educated and they are aware of most common symptoms (cough, fever and shortness of breath) [17]. A higher percentage of people who turned up for testing are employed $[19,20]$. There was a higher percentage turn up from participants with pre-existing health conditions compared to lower percentage turn up from participants with no pre-existing health conditions [18]. A higher percentage of people turned up in the category of participants who had positive previous experiences with the general quality of healthcare services $[24,25]$. 
Communication during the process of care delivery has a major influence on people's desire to seek healthcare and we see that reflected in this study as well. Those who received adequate communication after filling the form had a significant association with turning up for testing compared to those that did not receive any or incomplete communication [26,27]. Only the category of participants who get health information from verified sources like the NIMR website, Ministry of Health website, and radio or television had a higher percentage of participants that turned up for testing than those who did not.

Transportation challenge and location of test centres had an influence on the turn up of participants for testing. People who live in rural local government areas were less likely to turn up for testing, yet as reflected in the motivation for coming to test for COVID-19, they wanted to know their status and to seize the opportunity of the free testing.

Our study concludes that age, state of residence, type of LGA one resides, preexisting health conditions, knowledge of COVID-19 symptoms, previous healthcare experience, and type of and frequency of communication influence care seeking behavior of Nigerian citizens, specifically during COVID-19.

\section{Recommendations}

The study identified a range of areas for attention in relation to intervention and research. Based on the findings, there is a significant difference in the rate of utilization of healthcare services in Nigeria among different categories of people. Proximity to testing centers seemed to impact service utilization and so, accurate placement of testing centres needs to be considered. Inclusive systems in healthcare facilities should undergo reforms so that they can serve broader categories of people (such as female, unemployed, and old population) as we see in this case that utilization was not even between gender and age groups. It is critical to ensure knowledge and awareness of signs and symptoms of a disease to increase uptake of healthcare services and utilization of facilities. To further encourage utilization of healthcare facilities during any pandemic, adequate communication through verified ways like the Ministry of Health website, and radio/television should be employed to influence people's desire to seek healthcare services. Further research is needed to find out several factors influencing behaviour of people who live in across different type LGAs to seek healthcare services.

\section{Acknowledgement}

This study was made possible by the support and leadership of Nigeria Institute of Medical Research (NIMR), LifeBank Nigeria, CcHUB Nigeria Public Health Practice, CcHUB Design Lab Kigali, CcHUB Community Team and CcHUB Design Trainees in Nigeria.

\section{Bibliography}

1. World Health Organization. Situation Report 118 (2020).

2. National Academies of Sciences, Engineering, and Medicine. "Health-Care Utilization as a Proxy in Disability Determination". Washington, DC: The National Academies Press (2018).

3. Fein R. "On Achieving Access and Equity in Health Care". Milbank Quarterly 83.4 (2005): 157-190.

4. Geense WW., et al. "Barriers, facilitators and attitudes influencing health promotion activities in general practice: an explorative pilot study". BMC Family Practice 14.20 (2013).

5. Panda B and Thakur HP. "Decentralization and health system performance - a focused review of dimensions, difficulties, and derivatives in India”. BMC Health Service Research 16 (2016): 1-14.

6. Setia MS. "Methodology Series Module 3: Cross-sectional Studies”. Indian Journal of Dermatology 61.3 (2016): 261-264.

7. Centre for Disease Control and Prevention (CDC). Symptoms of Coronavirus (2020).

8. WHO (World Health Organization). Health impact assessment (HIA), determinants of health. Geneva, Switzerland: WHO (2017).

9. ODPHP. Healthy People 2020 topics and objectives: Disability and health. Washington, DC: US Department of Health and Human Services (2017b).

10. Thomas-Henkel C and M Schulman. "Screening for social determinants of health in populations with complex needs: Implementation considerations". Washington, DC: Center for Health Care Strategies, Inc (2017).

11. Salganicoff A., et al. "Women and health care in the early years of the Affordable Care Act: Key findings from the 2013 Kaiser women's health survey". Menlo Park, CA: Kaiser Family Foundation (2014).

12. Chan C., et al. "Factors affecting uptake of cervical and breast cancer screening among perimenopausal women in Hong Kong". Hong Kong Medical Journal 8 (2002): 334-341. 
13. Pronyk PM., et al. "Assessing health seeking behaviour among tuberculosis patients in rural South Africa". The International Journal of Tuberculosis and Lung Disease 5.7 (2001): 619-627.

14. NCHS. Health, United States. In Health, United States, 2016: With chartbook on long-term trends in health. Hyattsville (MD): National Center for Health Statistics (US) (2017b).

15. Pignatelli S., et al. "Factors predicting uptake of voluntary counselling and testing in a real-life setting in a mother-andchild center in Ouagadougou, Burkina Faso". Tropical Medicine and International Health 11 (2006): 350-357.

16. Ukwaja KN., et al. "Healthcare-seeking behavior, treatment delays and its determinants among pulmonary tuberculosis patients in rural Nigeria: a cross-sectional study". BMC Health Services Research 13.25 (2013).

17. Borges ALV., et al. "Women's reproductive health knowledge, attitudes and practices in relation to the Zika virus outbreak in northeast Brazil". PLoS ONE 13.1 (2013): e0190024.

18. O'Sullivan T and Bourgoin M. "Vulnerability in an Influenza Pandemic: Looking Beyond Medical Risk". Funded by the Public Health Agency of Canada (2010).

19. McKee-Ryan FM., et al. "Psychological and physical well-being during unemployment: A meta-analytic study". Journal of Applied Psychology 90.1 (2005): 53-76.

20. Thomas KC and Ellis AR. "Patterns of healthcare use and employment among people with disabilities". Disability and Health Journal 6.2 (2013): 133-140.

21. Li B., et al. "Prevalence and impact of cardiovascular metabolic diseases on COVID-19 in China". Clinical Research in Cardiology 109.5 (2020): 531-538.

22. Yang J., et al. "Prevalence of comorbidities and its effects in patients infected with SARS-CoV-2: a systematic review and meta-analysis". International Journal of Infectious Diseases 94 (2020): 91-95.

23. Onder,G., et al. "Case-Fatality Rate and Characteristics of Patients Dying in Relation to COVID-19 in Italy". JAMA 323.18 (2020): 1775-1776.

24. Latunji 00 and Akinyemi 00. "Factors influencing HealthSeeking Behaviour among Civil Servants in Ibadan, Nigeria". Annals of Ibadan Postgraduate Medicine 16.1 (2018): 52-60.
25. Centre for Disease Control (CDC). Severe Outcomes Among Patients with Coronavirus Disease 2019 (COVID-19) - United States, February 12-March 16, 2020. Morbidity Mortality Weekly Report (2020): 69.

26. Assessing competence in communication and interpersonal skills: the Kalamazoo II report (2020).

27. Bientzle M., et al. "The impact of physicians' communication styles on evaluation of physicians and information processing: A randomized study with simulated video consultations on contraception with an intrauterine device". Health Expectations 20.5 (2017): 845-851.

28. Osamor P and Grady C. "Factors associated with women's health care decision-making autonomy: empirical evidence from Nigeria". Journal of Biosocial Science 50 (2018): 70-85.

29. Senarath U and Gunawardena NS. "Women's autonomy in Decision Making for Health Care in South Asia”. Asia Pacific Journal of Public Health 21 (2009): 137-143.

\section{Assets from publication with us}

- Prompt Acknowledgement after receiving the article

- Thorough Double blinded peer review

- Rapid Publication

- Issue of Publication Certificate

- High visibility of your Published work

Website: https://www.actascientific.com/

Submit Article: https://www.actascientific.com/submission.php

Email us: editor@actascientific.com

Contact us: +919182824667 